\title{
Ocorrência de triatomíneos em ambientes silvestres e domiciliares do município de Manaus, Estado do Amazonas
}

\author{
Occurrences of triatomines in wild and domestic environments \\ in the municipality of Manaus, State of Amazonas
}

\author{
Nelson Ferreira Fé1, Laylah Kelre Magalhães ${ }^{1,2}$, Flávio Augusto Fé1, \\ Silvana Karina Arakian', Wuelton Marcelo Monteiro ${ }^{1,2,3}$ \\ e Maria das Graças Vale Barbosa ${ }^{1,2,4}$
}

\begin{abstract}
RESUMO
A análise da distribuição de triatomíneos é essencial para a formulação de estratégias de controle da doença de Chagas na Região Amazônica. Neste trabalho, apresentam-se os resultados de capturas realizadas em ambientes silvestres e artificiais, em localidades rurais e urbanas de Manaus, Amazonas. Dos 115 triatomíneos coletados, 85 (73,9\%) são da espécie Rhodnius pictipes, 25 (21,7\%) de Rhodnius robustus e cinco (4,4\%) de Panstrongylus geniculatus. A taxa de infecção natural por flagelados foi de 5,9\% para Rhodnius pictipes e 8\% para Rhodnius robustus. Nenhum exemplar de Panstrongylus geniculatus estava infectado. Todos os exemplares infectados eram oriundos de fragmentos de matas da zona urbana. Verificou-se que 106 (92,2\%) triatomíneos foram coletados no ambiente silvestre e nove (7,8\%) nas unidades domiciliares pela busca ativa. 0 gênero Rhodnius predominou nitidamente no ambiente silvestre. Os exemplares de Panstrongylus geniculatus, todos adultos, foram coletados no intradomicílio. Não foram encontrados indícios de colonização domiciliar por triatomíneos.
\end{abstract}

Palavras-chaves: Rhodnius pictipes. Rhodnius robustus. Panstrongylus geniculatus. Estado do Amazonas.

\begin{abstract}
Analysis of the distribution of triatomines is essential for formulating control strategies for Chagas disease in the Amazon region. In this paper, the results from trapping in wild and artificial environments in rural and urban localities in Manaus, Amazonas, are presented. Out of the 115 triatomines collected, 85 (73.9\%) were of the species Rhodnius pictipes, 25 (21.7\%) of Rhodnius robustus and five (4.4\%) of Panstrongylus geniculatus. The rate of natural infection by flagellates was 5.9\% for Rhodnius pictipes and $8 \%$ for Rhodnius robustus. None of the specimens of Panstrongylus geniculatus were infected. All of the infected specimens were from forest fragments in the urban zone. It was found that 106 insects (92.2\%) were collected from the forest environment and nine (7.8\%) in households, by means of the active search. The genus Rhodnius clearly predominated in the wild environment. The specimens of Panstrongylus geniculatus (all adults) were collected inside homes. There were no signs of domestic colonization by triatomines.
\end{abstract}

Key-words: Rhodnius pictipes. Rhodnius robustus. Panstrongylus geniculatus. State of Amazonas.

Por muito tempo considerada hipoendêmica na Região Amazônica, a doença de Chagas recentemente vem sendo reconhecida como um problema emergente e negligenciado nesta região ${ }^{17}$. 0 Inquérito Sorológico $\mathrm{Nacional}^{3}$ estimou uma prevalência de 1,9\% pra o Amazonas, o que proporciona um número esperado de 8.195 casos positivos para uma população de 435.379 habitantes à época. Recentemente, um inquérito realizado na área rural de Manaus detectou uma taxa de 1,3\% de indivíduos com sorologia positiva para a infecção pelo

1. Gerência de Entomologia, Fundação de Medicina Tropical do Amazonas, Manaus, AM. 2. Programa de Pós-graduação em Medicina Tropical, Universidade do Estado do Amazonas, Manaus, AM. 3. Departamento de Saúde Coletiva, Universidade Federal do Amazonas, Manaus, AM. 4. Centro Universitário Nilton Lins, Manaus, AM.

Endereço para correspondência: Prof. Wuelton Marcelo Monteiro. Pós-Graduação em Medicina Tropical/UEA. Av. Pedro Teixeira 25, Dom Pedro, 69040-000 Manaus, AM.

Fax: 5592 3656-8269

e-mail: wueltonmm@ibest.com.br

Recebido para publicação em 17/08/2009

Aceito em 09/11/2009
Trypanosoma cruzi $i^{9}$. 0 encontro desta prevalência é importante, adicionada ao fato de que foi registrada a presença de triatomíneos infectados invadindo domicílios, neste município ${ }^{14}$. Informações do Ministério da Saúde ${ }^{13}$ indicam que de 2005 a maio 2009 foram notificados 455 casos de doença de Chagas aguda no Brasil, dos quais 389 (85,5\%) ocorreram na Região Norte do país. Neste período, os estados brasileiros com maior número de casos registrados foram o Pará, com 310 (68,1\%) casos e o Amazonas, com $29(6,4 \%)^{13}$.

Pelo menos 18 espécies de triatomíneos silvestres têm sido encontradas na Região Amazônica, das quais 10 com registro de infecção por flagelados do tipo Trypanosoma cruzi $i^{2}{ }^{1112}$, demonstrando que a análise da distribuição de vetores de Trypanosoma cruzi é uma necessidade operativa para a formulação de estratégias de controle da doença de Chagas na região. No presente trabalho, apresentam-se os resultados preliminares de capturas de triatomíneos realizadas em ambientes silvestres e artificiais em diversas localidades rurais e urbanas de Manaus, Amazonas. 


\section{MATERIAL E MÉTODOS}

Área de estudo. As coletas de triatomíneos foram realizadas em ambientes silvestres e domiciliares de diversas localidades da zona rural e urbana do município de Manaus $\left(03^{\circ} 08^{\prime} 07^{\prime \prime}\right.$, $60^{\circ} 01^{\prime} 34^{\prime \prime} 0$ ), Estado do Amazonas (Figura 1). Na área rural, as coletas foram feitas em localidades adjacentes às rodovias BR-174, que liga Manaus a Boa Vista (RR), e AM-010, que vai de Manaus à Itacoatiara. Na área urbana, as localidades foram selecionadas devido à manutenção de fragmentos de mata na proximidade das residências, o que permitiu capturas tanto em ambientes silvestres, representado por encraves de mata mantidos no perímetro urbano, quanto artificiais. 0 ambiente silvestre, neste estudo, é composto por matas primárias geralmente modificadas por alteração antrópica devido às atividades de extrativismo de madeira e derrubada para o estabelecimento de culturas agrícolas. 0 ambiente artificial, por sua vez, é representado pela unidade domiciliar, composta pela residência, nas localidades urbanas, e pela residência e anexos (abrigos de animais domésticos, paióis de mantimentos e montes de telha e lenha), na zona rural. A distância entre as residências e 0 ambiente silvestre variava de 50 a 200 metros, dependendo da localidade. 0 clima do município é tropical chuvoso e úmido, com temperatura média de $26^{\circ} \mathrm{C}$.

Metodologia. As capturas foram realizadas pela equipe da Gerência de Entomologia da Fundação de Medicina Tropical do Amazonas (GE/FMTAM), de novembro de 2004 a agosto de 2005, que foi o período de duração de um projeto desta gerência. No ambiente silvestre de cada localidade, as coletas foram feitas durante cinco dias mensais consecutivos, com armadilhas do tipo Noireau ${ }^{15}$, usando camundongos como isca. Em cada localidade foram instaladas 24 armadilhas, distribuídas em oito palmeiras (três em cada). As armadilhas eram inspecionadas diariamente. No ambiente artificial, as capturas deram-se por buscas ativas não sistemáticas nas casas e anexos peridomiciliares, o que impossibilitou a determinação das taxas de infestação das unidades domiciliares. Na Comunidade São João, os triatomíneos foram coletados apenas por buscas ativas nas residências e anexos. Os exemplares coletados foram encaminhados à GE/FMTAM, onde se realizou a identificação específica e a verificação da taxa de infecção, pelo exame do conteúdo intestinal após compressão abdominal e dissecação em solução salina, buscando-se flagelados semelhantes ao Trypanosoma cruzi.

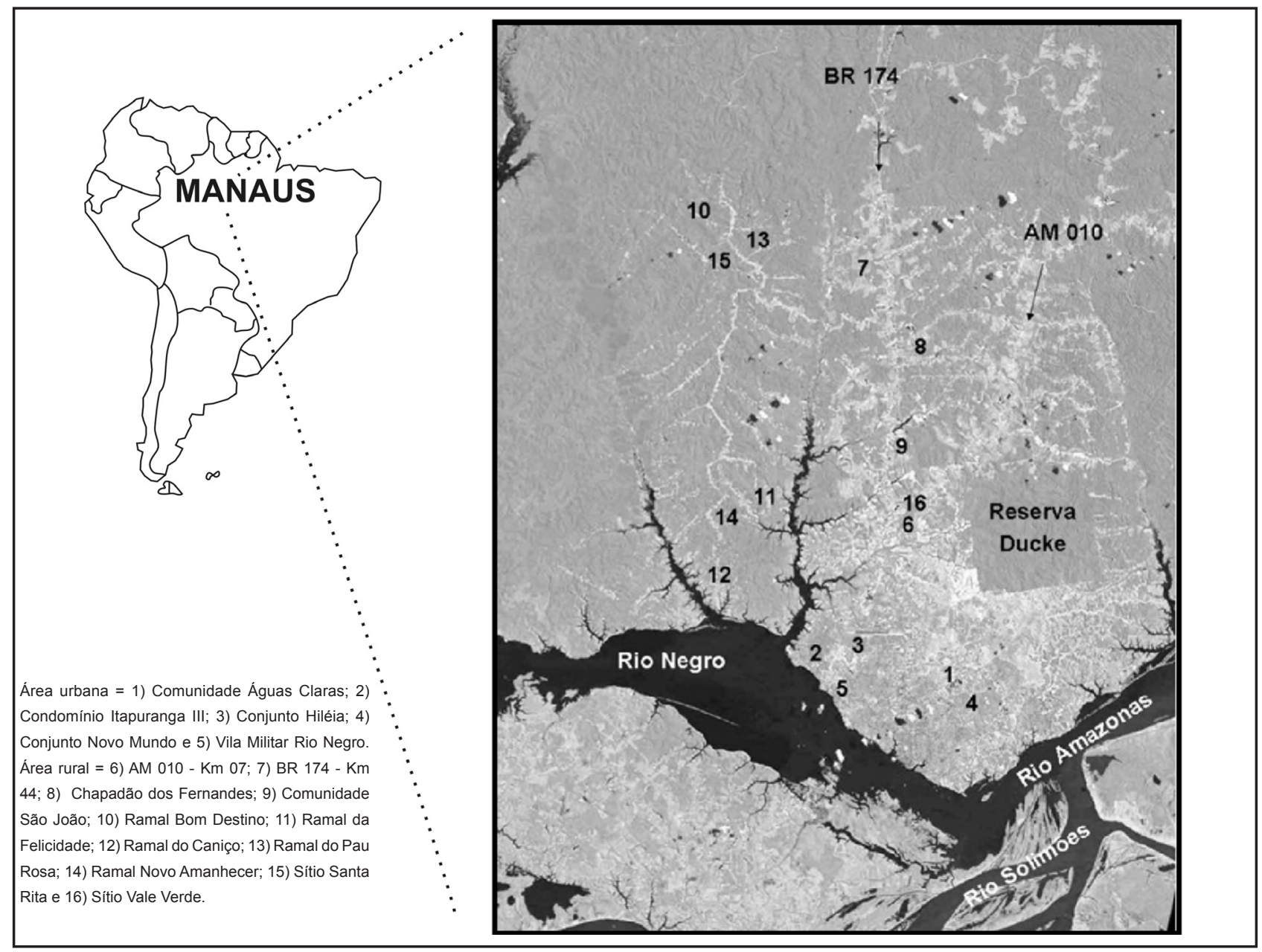

FIGURA 1

Imagem Landsat de Manaus, com a localização das áreas urbanas e rurais, onde foram coletados os triatomíneos. 


\section{RESULTADOS}

Foram coletados 115 exemplares de triatomíneos, distribuídos em três espécies. 0 Rhodnius pictipes, com 85 exemplares (53 ninfas e 32 adultos), correspondeu a 73,9\% do total coletado. As outras espécies coletadas foram Rhodnius robustus, com 25 (21,7\%) exemplares (15 ninfas e 10 adultos), e Panstrongylus geniculatus, com cinco (4,4\%) espécimes adultos (Tabela 1). No ambiente domiciliar foram encontrados apenas exemplares adultos, enquanto no ambiente silvestre foram observados exemplares de todos os estádios evolutivos.

A taxa de infecção natural por flagelados semelhantes ao Trypanosoma cruzi foi de 6,1\% no total. Observaram-se cinco (5,9\%) exemplares de Rhodnius pictipes infectados por flagelados, sendo três machos e duas fêmeas. Dois (8\%) exemplares fêmeas de Rhodnius robustus apresentaram infecção por flagelados. Nenhum exemplar de Panstrongylus geniculatus estava infectado (Tabela 1). Todos os exemplares infectados eram oriundos de fragmentos de matas da zona urbana.
Coletaram-se 84 (73\%) exemplares de triatomíneos na zona urbana e 31 (27\%) na zona rural. Rhodnius pictipes (83,5\%) e Panstrongylus geniculatus $(60 \%)$ predominaram na zona urbana, enquanto Rhodnius robustus foi mais abundante na zona rural (60\%). Na zona urbana, as localidades com maior número de triatomíneos coletados foram o Condomínio Itapuranga III, com 59 (51,3\%) exemplares e a Comunidade Águas Claras, com $21(18,3)$ exemplares. A localidade rural com maior número de triatomíneos coletados foi o Ramal da Felicidade, no Assentamento Tarumã Mirim, com 13 (11,3\%) exemplares, sendo 12 ninfas de uma única colônia de Rhodnius pictipes encontrada em ambiente silvestre, seguido pelo Chapadão dos Fernandes e pelo Sítio Vale Verde, com 3 (2,6\%) exemplares cada (Tabela 2).

Quanto ao ambiente de captura, verificou-se que 106 (92,2\%) triatomíneos foram coletados no ambiente silvestre e nove $(7,8 \%)$ nas unidades domiciliares pela busca ativa. As três espécies foram coletadas nos domicílios. Porém, Rhodnius pictipes e Rhodnius robustus predominaram nitidamente no ambiente silvestre, com 97,6 e 92\% dos exemplaress coletados para cada

TABELA 1

Estádios evolutivos e taxas de infecção por flagelados das espécies de triatomíneos coletadas no município de Manaus, no período de novembro de 2004 a agosto de 2005.

\begin{tabular}{|c|c|c|c|c|c|c|c|c|c|c|}
\hline \multirow[b]{2}{*}{ Espécie } & \multicolumn{6}{|c|}{ Estádio } & \multicolumn{2}{|c|}{ Taxa de infecção } & \multicolumn{2}{|c|}{ Total } \\
\hline & I & II & III & IV & V & adulto & $\mathrm{n}^{0}$ & $\%$ & $\mathrm{n}^{0}$ & $\%$ \\
\hline Rhodnius pictipes & 5 & 13 & 11 & 14 & 10 & 32 & 5 & 5,9 & 85 & 73,9 \\
\hline Rhodnius robustus & 3 & 2 & 1 & 4 & 5 & 10 & 2 & 8,0 & 25 & 21,7 \\
\hline Panstrongylus geniculatus & 0 & 0 & 0 & 0 & 0 & 5 & 0 & 0,0 & 05 & 4,4 \\
\hline Total & 8 & 15 & 12 & 18 & 15 & 47 & 7 & 6,1 & 115 & 100,0 \\
\hline
\end{tabular}

TABELA 2

Triatomíneos coletados no município de Manaus, Estado do Amazonas, no período de novembro de 2004 a agosto de 2005, conforme localidades e ambientes de coleta.

\begin{tabular}{|c|c|c|c|c|c|c|c|c|c|c|c|}
\hline \multirow[t]{2}{*}{ Localidade } & \multicolumn{3}{|c|}{ Rhodnius pictipes } & \multicolumn{3}{|c|}{ Rhodnius robustus } & \multicolumn{3}{|c|}{ Panstrongylus geniculatus } & \multicolumn{2}{|c|}{ Total } \\
\hline & $\mathrm{AD}$ & AS & total & $\mathrm{AD}$ & AS & total & $\mathrm{AD}$ & AS & total & $n^{0}$ & $\%$ \\
\hline \multicolumn{12}{|l|}{ Zona urbana } \\
\hline Comunidade Águas Claras & 0 & 17 & 17 & 1 & 3 & 4 & 0 & 0 & 0 & 21 & 18,3 \\
\hline Condomínio Itapuranga III & 1 & 52 & 53 & 1 & 5 & 6 & 0 & 0 & 0 & 59 & 51,3 \\
\hline Conjunto Hiléia & 0 & 0 & 0 & 0 & 0 & 0 & 1 & 0 & 1 & 1 & 0,8 \\
\hline Conjunto Novo Mundo & 0 & 0 & 0 & 0 & 0 & 0 & 2 & 0 & 2 & 2 & 1,7 \\
\hline Vila Militar Rio Negro & 1 & 0 & 1 & 0 & 0 & 0 & 0 & 0 & 0 & 1 & 0,8 \\
\hline Subtotal & 2 & 69 & 71 & 2 & 8 & 10 & 2 & 1 & 3 & 84 & 73,0 \\
\hline \multicolumn{12}{|l|}{ Zona rural } \\
\hline AM010 - Km 07 & 0 & 0 & 0 & 0 & 0 & 0 & 1 & 0 & 1 & 1 & 0,8 \\
\hline BR174 - Km 44 & 0 & 1 & 1 & 0 & 0 & 0 & 0 & 0 & 0 & 1 & 0,8 \\
\hline Chapadão dos Fernandes & 0 & 3 & 3 & 0 & 0 & 0 & 0 & 0 & 0 & 3 & 2,6 \\
\hline Comunidade São João & 0 & 0 & 0 & 0 & 1 & 1 & 1 & 0 & 1 & 2 & 1,7 \\
\hline Ramal Bom Destino & 0 & 1 & 1 & 0 & 0 & 0 & 0 & 0 & 0 & 1 & 0,8 \\
\hline Ramal da Felicidade & 0 & 1 & 1 & 0 & 12 & 12 & 0 & 0 & 0 & 13 & 2,6 \\
\hline Ramal do Caniço & 0 & 1 & 1 & 0 & 1 & 1 & 0 & 0 & 0 & 2 & 1,7 \\
\hline Ramal do Pau Rosa & 0 & 1 & 1 & 0 & 0 & 0 & 0 & 0 & 0 & 1 & 0,8 \\
\hline Ramal Novo Amanhecer & 0 & 1 & 1 & 0 & 1 & 1 & 0 & 0 & 0 & 2 & 1,7 \\
\hline Sítio Santa Rita & 0 & 2 & 2 & 0 & 0 & 0 & 0 & 0 & 0 & 2 & 1,7 \\
\hline Sítio Vale Verde & 0 & 3 & 3 & 0 & 0 & 0 & 0 & 0 & 0 & 3 & 2,6 \\
\hline Subtotal & $\mathbf{0}$ & 14 & 14 & $\mathbf{0}$ & 15 & 15 & 2 & $\mathbf{0}$ & 2 & 31 & 27,0 \\
\hline Total & 2 & 83 & 85 & 2 & 23 & 25 & 5 & 0 & 5 & 115 & 100,0 \\
\hline
\end{tabular}

AD: ambiente domiciliar, AS: ambiente silvestre. 
espécie, respectivamente. Todos os exemplares de Panstrongylus geniculatus são provenientes do ambiente domiciliar. No geral, a frequência dos triatomíneos no ambiente domiciliar foi de 7,1\% na zona urbana e $6,5 \%$ na zona rural.

Dos 83 exemplares de Rhodnius pictipes coletados no ambiente florestal, 58 (69,9\%) foram capturados na palmeira Maximiliana maripa, 23 (27,7\%) em Oenocarpus bacaba e dois (2,4\%) em Oenocarpus bataua, enquanto os 23 exemplares de Rhodnius robustus deste tipo de ambiente predominaram em Maximiliana maripa, com 19 (82,6\%) exemplares, seguida por Oenocarpus bacaba, com quatro (17,4\%).

\section{DISCUSSÃo}

As espécies Rhodnius pictipes, Rhodnius robustus e Panstrongylus geniculatus já foram registradas anteriormente no município de Manaus ${ }^{101114}$. Estas espécies são frequentemente encontradas com altas taxas de infecção natural pelo Trypanosoma cruzi em diversos ecótopos naturais na Amazônia Brasileira, onde esporadicamente invadem as residências ${ }^{1}$. A baixa diversidade de espécies encontrada na maioria dos trabalhos realizados no Amazonas $^{6101114}$, corroborada pela presente investigação, pode ser devida à metodologia empregada na captura dos triatomíneos, em ambientes com fragmentação das florestas nativas por ação antrópica, não abrangendo ecótopos como tocas e abrigos de diferentes espécies de animais silvestres.

Rhodnius pictipes, que foi a espécie predominante neste trabalho, é um triatomíneo silvestre de ampla distribuição na América do Sul. Tem sido encontrada no Brasil nos Estados do Acre, Amazonas, Maranhão, Mato Grosso, Mato Grosso do Sul, Pará, Piauí e Tocantins ${ }^{819}$. Esta espécie foi encontrada naturalmente infectada pelo Trypanosoma cruzi, pelo Trypanosoma rangeli e com infecção mista $^{416}$. Neste trabalho, prevaleceu em fragmentos florestais da área urbana, com baixa frequiência nos domicílios. No município de São Paulo de Olivença, na região do Alto Solimões, onde foi registrado o primeiro caso de doença de Chagas do Estado do Amazonas, Rhodnius pictipes foi a espécie predominante no intradomicílio ${ }^{6}$.

A segunda espécie em número de exemplares foi Rhodnius robustus, que prevaleceu na zona rural. Esta espécie é considerada de importância secundária na transmissão do Trypanosoma cruzi $i^{6}$. Porém, neste trabalho apresentou a maior taxa de infecção por flagelados, apesar da baixa freqüência nos domicílios. Na Venezuela, demonstrou-se que Rhodnius robustus invade residências e se alimenta em humanos ${ }^{7}$. A baixa taxa de infestação domiciliar por esta espécie sugere que os exemplares abandonam as casas depois da hematofagia ou morrem sem se reproduzir no interior das mesmas ${ }^{7}$.

Panstrongylus geniculatus, apesar de coletada em pequeno número e sem infecção natural por flagelados, foi encontrada apenas no ambiente artificial, mais especificamente no intradomicílio. Esta espécie é um importante vetor silvestre ${ }^{8}$, que invade esporadicamente as residências ${ }^{12} 1420$ atraída pela luz ${ }^{12}$ e coloniza abrigos de suínos construídos na proximidade ou contíguos com as habitações humanas no Estado do Paráa ${ }^{20}$. Nesse contexto, pode contribuir na transmissão domiciliar da doença de Chagas, já que em algumas áreas tem se apresentado com elevadas taxas de infecção natural ${ }^{5}$.

0 predomínio dos triatomíneos na zona urbana sugere que os fragmentos florestais que persistem em algumas localidades permitem a manutenção do ciclo enzoótico de Trypanosoma cruzi, evidenciando risco de infecção para a população que vive na proximidade destas áreas. Nestes locais, são frequentes palmeiras das espécies Maximiliana maripa, Oenocarpus bacaba e Oenocarpus bataua nas áreas de vegetação secundária e áreas desmatadas nos terrenos baixos não inundáveis, inclusive nos quintais das casas, onde se refugiam roedores e marsupiais como Didelphis marsupialis e outros hospedeiros de Trypanosoma cruzi.

Mesmo considerando-se que a transmissão da doença de chagas na Região Amazônica ocorra em grande parte dos casos por via oral, é necessária a presença de um vetor infectado muito próxima do homem, para que ela aconteça ${ }^{18}$. A localização das residências nas proximidades dos ecótopos naturais dos triatomíneos possibilita a invasão esporádica dos domicílios por exemplares adultos, como observado neste trabalho para Rhodnius pictipes, Rhodnius robustus e Panstrongylus geniculatus. 0 desmatamento, a redução dos reservatórios silvestres, a proliferação de mamíferos oportunistas (roedores e marsupiais) nos ambientes degradados e a introdução da luz elétrica também são fatores potencialmente relacionados com a ocorrência da doença nesta região ${ }^{1}$. Destaca-se que não existe nenhum programa sistemático de vigilância entomológica instituído nas localidades estudadas.

Concluindo, verificou-se a presença de Rhodnius pictipes, Rhodnius robustus e Panstrongylus geniculatus em localidades rurais e urbanas do município de Manaus, com taxas de infecção natural por flagelados relativamente baixas em relação aos trabalhos anteriormente desenvolvidos na Amazônia ${ }^{1}$. Rhodnius pictipes predominou nos fragmentos florestais da zona urbana, enquanto Rhodnius robustus prevaleceu nas matas da zona rural e Panstrongylus geniculatus foi coletada exclusivamente no intradomicílio. Não foram encontrados indícios de colonização domiciliar por triatomíneos. Na continuação deste estudo, planejase capturar um maior número de triatomíneos, estendendo as investigações para outras localidades e outros tipos de ecótopos, bem como realizar o isolamento de amostras de Trypanosoma cruzi para posterior caracterização biológica e molecular.

\section{AGRADECIMENTOS}

Aos funcionários da Fundação de Medicina Tropical do Amazonas que cooperaram direta ou indiretamente na execução dos trabalhos de campo que originara este manuscrito.

\section{REFERÊNCIAS}

1. Aguilar HM, Abad-Franch F, Dias JCP, Junqueira ACV, Coura JR. Chagas disease in the Amazon Region. Memórias do Instituto Oswaldo Cruz 102: 47-55, 2007.

2. Barrett TV, Guerreiro JCH. Os triatomíneos (Hemiptera, Reduviidae) em relação à doença de Chagas na Amazônia. In: Val AL, Figliuolo R, Feldberg E (eds) Bases 
Científicas para Estratégia de Preservação e Desenvolvimento da Amazônia: Fatos e Perspectivas. Instituto Nacional de Pesquisas da Amazônia, Manaus, p.119-130, 1991

3. Camargo ME, Silva GR, Castilho EA, Silveira AC. Inquérito sorológico da prevalência da infecção chagásica no Brasil, 1975-1980. Revista do Instituto de Medicina Tropical de São Paulo 26: 192-204, 1984

4. Carcavallo RU, Martinez-Silva R, Otero MAA, Tonn RJ. Infección natural de Rhodnius robustus Larrouse y Rhodnius pictipes Stal por T. cruzi y T. rangeli en Venezuela. Boletín de la Dirección de Malariología y Saneamiento Ambiental 15:117-120, 1975.

5. Carrasco HJ, Torrellas A, García C, Segovia M, Feliciangeli MD. Risk of Trypanosoma cruzi I (Kinetoplastida: Trypanosomatidae) transmission by Panstrongylus geniculatus (Hemiptera: Reduviidae) in Caracas (Metropolitan District) and neighboring States, Venezuela. International Journal for Parasitology 35: 1379-1384, 2005.

6. Fé NF, França MS, Carvalho-Costa FA. Reassessing the entomological investigation around the first autochthonous case of Chagas disease in Western Brazilian Amazon. Memórias do Instituto Oswaldo Cruz 104: 121-123, 2009.

7. Feliciangeli MD, Dujardin JP, Bastrenta B, Mazzarri M, Villegas J, Flores M, Muñoz M. Is Rhodnius robustus (Hemiptera: Reduviidae) responsible for Chagas disease transmission in Western Venezuela? Tropical Medicine and International Health 7: 280-287, 2002

8. Lent H, Wygodzinsky P. Revision of the Triatominae (Hemiptera, Reduviidae), and their significance as vectors of Chagas'disease. Bulletin of the American Museum of Natural History 163: 123-520, 1979.

9. Magalhães BML. Doença de Chagas no Amazonas: Prevalência sorológica em área rural de Manaus. Dissertação de Mestrado, Universidade do Estado do Amazonas, Manaus, AM, 2009.

10. Magalhães LKC, Prestes SR, Fé NF, Ciriano CM, Fé FAA, Fabiano MP, Maciel MG, Oliveira JC, Silveira H, Barbosa MGV. Infecção natural e diversidade de espécies de vetores de doença de Chagas nos municípios de Manaus, Coari e Tefé/AM. Revista da Sociedade Brasileira de Medicina Tropical 42: 156, 2009.

11. Miles MA, Arias JR, Souza AA. Chagas' disease in the Amazon Basin: V. Periurban palms as habitats of Rhodnius robustus and Rhodnius pictipes -
Triatomine vectors of Chagas' disease. Memórias do Instituto Oswaldo Cruz 78: 391-398, 1983

12. Miles MA, Souza AA, Povoa M. Chagas disease in the Amazon Basin. III - Ecotopes of ten triatomine bug species (Hemiptera, Reduviidae) from the vinicity of Belém, Pará State, Brazil. Journal of Medical Entomology 18:266-278, 1981.

13. Ministério da Saúde. Casos de Doença de Chagas Aguda (DCA). Brasil, Grandes Regiões e Unidades Federadas, 2005 a 2009. Disponível em http://portal.saude. gov.br/portal/arquivos/pdf/tabchagascasos0509.pdf (acessado em 05/10/09).

14. Naiff MF, Naiff RD, Barrett TV. Vetores selváticos de doença de Chagas na área urbana de Manaus (AM): atividade de vôo nas estações secas e chuvosas. Revista da Sociedade Brasileira de Medicina Tropical 31: 103-105, 1998.

15. Noireau F, Abad-Franch F, Valente SA, Dias-Lima A, Lopes CM, Cunha V, Valente V, Palomeque F, Carvalho-Pinto CJ, Sherlock I, Aguilar M, Steindel M, Grisard E, Jurberg J. Trapping triatominae in silvatic habitats. Memórias do Instituto Oswaldo Cruz 97: 61-63, 2002.

16. Otero AMA, Carcavallo RU, Tonn RJ. Notas sobre la biología, ecología y distribuición geográfica de Rhodnius pictipes Stal, 1872 (Hemiptera Reduviidae). Bolletín de la Dirección de Malariología y Saneamiento Ambiental 16: 163-168, 1976.

17. Pan American Health Organization. Implementation of the Intergovernmenta Initiative for Surveillance and Control of Chagas Disease in the Amazon Region (AMCHA). Disponível em < http://www.paho.org/english/AD/DPC/CD/dch-amcha2004.pdf (acessado em 20/07/2005>)

18. Pinto AYN, Valente SA, Valente VC, Ferreira-Junior AG, Coura JR. Fase aguda da doença de Chagas na Amazônia brasileira. Estudo de 233 casos do Pará, Amapá e Maranhão observados entre 1988 e 2005. Revista da Sociedade Brasileira de Medicina Tropical 41: 602-614, 2008

19. Silveira AC, Feitosa VR, Borges R. Distribuição de triatomíneos capturados no ambiente domiciliar, no período de 1975/83, Brasil. Revista Brasileira de Malariologia e Doenças Tropicais 36: 15-312, 1984.

20. Valente VC, Valente SAS, Noireau F, Carrasco HJ, Miles MA. Chagas disease in the Amazon basin: association of Panstrongylus geniculatus (Hemiptera: Reduviidae) with domestic pigs. Journal of Medical Entomology 35: 99-103, 1998 